\title{
Recherche des facteurs responsables du déclenchement du tallage chez le ray-grass d'Italie (Lolium multiflorum lam.) en conditions naturelles
}

\author{
Jean-Claude SIMON \& Richard DELECOLLE $\left(^{*}\right)$
}

I.N.R.A., Station d'Agronomie, 4 rue Stang-Vihan, F 29000 Quimper.

* I.N.R.A., Station de Bioclimatologie, Centre de Recherches d'Avignon, F84140 Montfavet.

\begin{abstract}
Grâce à l'utilisation de la régression multiple et, notamment, de la « ridge regression » qui permet de protéger les résultats des instabilités dues à l'interdépendance des facteurs explicatifs, on montre que, d'un point de vue climatique, la phase levée-début tallage du Ray Grass d'Italie est déterminée, e, conditions naturelles, par le rayonnement global et la température de l'air et, à un degré moindre, par la température du sol. Mais la part de variance ainsi expliquée n'est que de 55 p. 100 , probablement en raison de la variabilité génétique importante du matériel utilisé. Une somme de température du sol de $284^{\circ} \mathrm{C}$. j entre la levée et début tallage, avec un seuil de $0{ }^{\circ} \mathrm{C}$ permet d'estimer la date de début tallage.
\end{abstract}

Mots clés additionnels : Microclimat, température, rayonnement, ridge regression.

\begin{abstract}
Determining factors for the beginning of tillering in Italian rye-grass (Lolium multiflorum lam.) under field conditions.

The relationship between climate and time from emergence to tillering in Italian rye-grass under field conditions has been investigated through multiple regression. In order to protect the results from the instability which arises from high correlations between climatic predictors, ridge regression was used, with a ridge coefficient $\mathrm{k}=0.1$. Provided soil water is not limiting, the predictors with highest weight were first, radiation and air temperature then soil temperature, but only $50 \%$ of the variability could be explained in this way.

The field results were nevertheless consistent with the literature, which most often deals with experiments under controlled conditions.

Using daily soil temperature above $0{ }^{\circ} \mathrm{C}$, a temperature sum of 284 degree-days from emergence gave a good estimation of the beginning of tillering.
\end{abstract}

Additional key words : Microclimate, temperature, solar radiation, ridge regression.

\section{INTRODUCTION}

A l'issue de la phase semis-levée (SIMON, 1981b), le raygrass d'Italie commence une période de croissance végétative, généralement assez lente, qui se caractérise par l'apparition des feuilles successives du maître-brin. A l'aisselle de chacune de ces feuilles se forme un bourgeon de talle. Contrairemeǹt aux ébauches de feuilles, ce dernier n'entre pas immédiatement en croissance ; il prend le nom de talle que lorsqu'il apparaît à l'extérieur. Lorsque la $1^{\text {re }}$ talle se présente à la base de la $1^{\text {re }}$ feuille, on parle de début tallage.

Ce stade, qui s'accompagne de l'émission des premières racines de tallage, marque, pour la jeune plante, le début d'une période de moindre sensibilité au froid, la destruction de l'hypocotyle étant alors moins grave de conséquences pour la survie de celle-ci.

Or, la place du ray-grass d'Italie dans les systèmes fourragers intensifs (semis d'automne après maïs) conduit à des risques de gelée sur les jeunes plantules. Il est donc important de connaître le déterminisme climatique de la phase levée-début tallage; afin de fixer les limites de la date de levée et, par voie de conséquence, de la dáte de semis.

A partir de résultats déjà existants sur graminées fourragères, mais le plus souvent obtenus en conditions contrôlées, cet article se propose de rechercher, en conditions naturelles, les relations entre le déclenchement du tallage et les factcurs climatiques les plus aisément mesurables ou calculables. 
Cependant, s'il est aisé de caractériser en conditions contrôlées l'effet d'un facteur climatique isolé, l'analyse devient plus délicate dans le milieu naturel, où tous les facteurs mesurés sont corrélés entre eux. Ceci est particulièrement gênant quand on recherche, par des méthodes statistiques comme la régression multiple, les relations entre la plante et son milieu : c'est pourquoi, comparativement à la régression classique, nous avons utilisé une méthode qui semble mieux adaptée à cette situation: la « ridge regression».

\section{ANALYSE BIBLIOGRAPHIQUE}

A partir du stade levée, le ray-grass d'Italie traverse 2 phases successives qui se chevauchent tardivement. La $1^{\text {ro }}$ est caractérisée par la croissance des ébauches foliaires successives, chaque feuille engendrant un bourgeon de talle (GILLET, 1980) ; la $2^{e}$, par le début de la croissance de la $1^{\text {re }}$ talle, quand la plante a 4 feuilles visibles.

Ces deux périodes sont d'inégale longueur, la $1^{\text {rc }}$ étant de loin la plus longue (une croissance relativement faible de la $1^{\text {re }}$ talle étant suffisante pour qu'elle devienne visible).

D'après la bibliographie, les facteurs actifs du milieu sont différents suivant ces 2 périodes :

Le phyllochrone est essentiellement lié à la température, la loi d'action étant du type courbe en cloche classique (Durand, 1967), avec un optimum voisin de $25^{\circ} \mathrm{C}$ sur Lolium (COOPER, 1964). Les hauts niveaux d'éclairement accélèrent le rythme d'émission sur Lolium (BEEVERS \& EDWARDS, 1960), l'ombrage le ralentit (FRIEND et al., 1962). Les autres facteurs actifs, telle la compétition (GILLET, 1970), ne semblent pas devoir être ici retenus, compte tenu du stade de la plante. Selon Gillet (1970), seule la température doit être retenue pour son action à court terme sur la vitesse phyllochronique, les autres facteurs ne l'influençant qu'au début, de façon irréversible.

La croissance des talles est sous l'influence de l'éclairement. Les hauts niveaux d'éclairement sont favorables, à l'unanimité des auteurs. Les résultats relatifs aux autres facteurs sont par contre souvent contradictoires. BOMMER (1962) a constaté l'existence d'un optimum de température ; AUDA et al. (1966) ont montré l'action favorable de la nutrition azotée aux forts éclairements, l'effet étant sinon néfaste. GILLET (1970) donne une interprétation des phénomènes qui explique les contradictions entre auteurs. Le principal facteur limitant du tallage serait le niveau de glucides solubles disponibles: ce dernier explique le rôle prépondérant du rayonnement, l'effet favorable de températures alternées jour-nuit, l'action néfaste des faibles éclairements quand la fertilisation azotée est élevée.

En conclusion, cette brève analyse bibliographique montre que 2 facteurs doivent théoriquement jouer un rôle déterminant sur le déclenchement du tallage du ray-grass d'Italie: la température, par son action sur la vitesse phyllochronique, et le rayonnement, par son rôle sur la croissance des talles.

\section{MATÉRIEL ET MÉTHODE EXPÉRIMENTALE}

\section{A. Le milieu pédoclimatique}

Situé à Quimper et constitué d'un sol d'origine granitique appartenant au type argilo-limoneux, le champ d'expérience est sous l'influence d'un climat océanique, dont les caractéristiques moyennes sont données par SIMON (1979). Au cours des 3 années d'essai, le facteur hydrique n'a jamais été strictement limitant, puisque l'humidité du sol, dans l'horizon prospecté par les racines séminales $(0-10 \mathrm{~cm})$, n'a jamais été inférieure au point de flétrissement permanent ( $\mathrm{pF} 4.2$ correspond à une humidité pondérale de $10 \mathrm{p} .100$ pour le sol considéré).

\section{B. La plante}

C'est la variété diploïde de ray-grass d'Italie « Tiara » qui a servi de support à nos études. Comme pour toutes les graminées fourragères, c'est une variété synthétique, donc de variabilité génétique importante.

\section{La technique expérimentale}

L'observation des phases levée-début tallage est effectuée sur des semis échelonnés dans le temps, du mois d'avril au mois de novembre, donc dans des conditions climatiques très diversifiées. Les semis ont été répétés 3 années successives de 1977 à 1979. Les semis de 1979 ont été doublés : une série a été effectuée en conditions normales, l'autre subissant un arrosage hebdomadaire compensant l'E.T.P. (tabl. 1). Chaque semis se compose de 5 répétitions, chacune d'elles étant constituée par $1 \mathrm{~m}$ de rang de semis. Le semis est effectué à l'aide d'un semoir à 1 rang, à une profondeur de $1 \mathrm{~cm}$, pour un écartement entre rangs de $20 \mathrm{~cm}$. Le peuplement semé est voisin de 100 plantes au m linéaire.

\section{La technique de comptage}

Indiquons tout d'abord qu'un stade phénologique est considéré comme atteint quand $50 \mathrm{p} .100$ des plantes observées sont à ce stade. La date de réalisation du stade phénologique est donc fixée a posteriori quand tous les individus qui en ont eu la possibilité ont atteint ce stade.

Le stade levée correspond au moment où le coléoptile pointe hors du sol et le stade début tallage à l'instant où la $1^{\text {rc }}$ talle primaire apparaît à l'aisselle de la $1^{\text {re }}$ feuille. Les comptages sont effectués tous les 2 jours en moyenne, sur 5 fois $1 \mathrm{~m}$ linéaire de rang, par traitement.

La date 50 p. 100 de début tallage a été déterminée à partir des valeurs moyennes, sur les 5 répétitions, du nombre de plantes ayant une talle visible.

\section{Les mesures climatiques}

Sur chaque parcelle, on détermine la date des stades levée et début tallage. Pour la période séparant ces 2 dates, on détermine la moyenne ou la somme des valeurs journalières des 5 paramètres climatiques suivants :

- Rg, rayonnement global (en cal. $\mathrm{cm}^{-2} \cdot \mathrm{j}^{-1}$ ), calculé par la formule de BLACK,

- $\mathrm{T}_{\mathrm{a}}$, température de l'air sous abri à $1,50 \mathrm{~m}$,

$-T_{\mathrm{s}}$, température du sol sous le ray-grass à $-10 \mathrm{~cm}$,

$-D_{h}$, déficit hydrique théorique $=$ ETP-P $($ ETP étant l'évapotranspiration potentielle calculée par la formule de TURC et $\mathbf{P}$, les précipitations),

$-\mathrm{H}_{\mathrm{s}}$, humidité pondérale du sol pour l'horizon 0-10 $\mathrm{cm}$.

\section{Méthodes de régression utilisées}

L'utilisation dans la régression multiple de variables explicatives corrélées entre elles peut entraîner dans les résultats de celle-ci des perturbations allant jusqu'au nonsens (HOERL \& KENNARD, 1970).

En effet, la corrélation (colinéarité) entre variables explicatives crée des problèmes d'inversion de la matrice 
TABLEAU 1.

Dates de levée des semis observés au cours des trois campagnes de mesures. Emergence dates for the three years of study.

\begin{tabular}{|c|c|c|c|c|c|c|c|c|c|c|c|}
\hline 1977 & $\begin{array}{c}N^{0} \text { du semis } \\
\text { Date }\end{array}$ & $\begin{array}{c}1 \\
20 / 4\end{array}$ & $\begin{array}{c}2 \\
3 / 5\end{array}$ & $\begin{array}{c}3 \\
15 / 5\end{array}$ & $\begin{array}{c}4 \\
27 / 5\end{array}$ & $\begin{array}{c}5 \\
13 / 9\end{array}$ & $\begin{array}{c}6 \\
29 / 9\end{array}$ & $\begin{array}{c}7 \\
20 / 10\end{array}$ & $\begin{array}{c}8 \\
5 / 11\end{array}$ & & \\
\hline 1978 & $\begin{array}{c}\mathrm{N}^{\circ} \text { du semis } \\
\text { Date }\end{array}$ & $\begin{array}{c}9 \\
21 / 4\end{array}$ & $\begin{array}{l}10 \\
1 / 5\end{array}$ & $\begin{array}{c}11 \\
21 / 5\end{array}$ & $\begin{array}{c}12 \\
19 / 6\end{array}$ & $\begin{array}{c}13 \\
20 / 6\end{array}$ & & & & & \\
\hline $1979 \mathrm{sec}$ & $\begin{array}{c}\text { No du semis }^{\text {Date }}\end{array}$ & $\begin{array}{l}14 \\
2 / 5\end{array}$ & $\begin{array}{l}15 \\
7 / 5\end{array}$ & $\begin{array}{c}16 \\
14 / 5\end{array}$ & $\begin{array}{c}17 \\
22 / 5\end{array}$ & $\begin{array}{c}18 \\
23 / 5\end{array}$ & $\begin{array}{c}19 \\
12 / 6\end{array}$ & $\begin{array}{l}20 \\
4 / 8\end{array}$ & $\begin{array}{c}21 \\
15 / 8\end{array}$ & $\begin{array}{c}22 \\
12 / 9\end{array}$ & $\begin{array}{c}23 \\
4 / 10\end{array}$ \\
\hline 1979 irrigué & $\mathrm{N}^{\circ} \underset{\text { Date }}{\text { du semis }}$ & $\begin{array}{l}24 \\
2 / 5\end{array}$ & $\begin{array}{l}25 \\
7 / 5\end{array}$ & $\begin{array}{c}26 \\
13 / 5\end{array}$ & $\begin{array}{c}27 \\
18 / 5\end{array}$ & $\begin{array}{c}28 \\
23 / 5\end{array}$ & $\begin{array}{c}29 \\
12 / 6\end{array}$ & $\begin{array}{c}30 \\
29 / 7\end{array}$ & $\begin{array}{c}31 \\
16 / 8\end{array}$ & $\begin{array}{c}32 \\
11 / 9\end{array}$ & $\begin{array}{c}33 \\
2 / 10\end{array}$ \\
\hline
\end{tabular}

des corrélations entre variables et conduit à un vecteur de coefficients de régression entaché d'une grande variabilité et « trop long" en espérance (MARQUARDT \& SNEE, 1975). Les coefficients deviennent alors très grands en valeur absolue et sont susceptibles de changements intempestifs de signe pour des variations minimes dans les données.

Les désagréments consécutifs aux propriétés décrites cidessus sont analysés par DUBY \& MASSON (1980). On peut mesurer l'effet de la colinéarité de chaque variable $j$, vis-àvis des autres variables, en calculant un paramètre dénommé VIF par les auteurs anglo-saxons (Variance Inflation Factor) égal à $\frac{1}{1-R^{2} j}$ où $R j$ est le coefficient de corrélation multiple entre cette variable $\mathrm{j}$ et toutes les autres. On peut interpréter cette grandeur comme le rapport de la variance du coefficient estimé de la j-ième variable à ce qu'elle serait si les variables étaient indépendantes. On peut considérer que, pour qu'une équation de régression soit acceptable, VIF ne doit pas dépasser 2 pour chaque variable (soit une corrélation avec les autres variables de 0,7 ).

Pour essayer de pallier ces inconvénients qui sont la plaie des études de régression en agrométéorologie (KATZ, 1979), nous avons utilisé la technique de « ridge regression » (HoERL, 1962 ; MARQuARDT \& SNEE, 1975; DRAPER \& VAN NOSTRAND, 1979). Cette technique consiste à rajouter, à chaque terme diagonal de la matrice des corrélations, un coefficient $\mathrm{k}$, généralement compris entre 0 et 1 . Ce coefficient introduit un biais dans la solution des moindres carrés, mais conduit à un vecteur de coefficients de régression moins variable. Le problème consiste alors à trouver le coefficient " optimal » et plusieurs méthodes sont disponibles à cette fin (GIBBONS, 1981). Nous utiliserons ici la «trace ridge", qui est la représentation graphique des valeurs prises par les coefficients de régression pour diverses valeurs de $\mathrm{k}$. La valeur « optimale » de $\mathrm{k}$ est celle pour laquelle la variation se stabilise (MARQUARD \& SNEE, 1975). Pour la présente étude, nous avons utilisé une méthode de régression par introduction successive des variables explicatives offrant la possibilité d'introduire également un coefficient de ridge $\mathrm{k}$.

\section{RÉSULTATS}

\section{A. Analyse descriptive de la phase levée-début tallage}

L'apparition de la $1^{\text {re }}$ talle primaire à l'aisselle de la $1^{\text {re }}$ feuille se produit le plus souvent sur des plantes au stade 4 feuilles ( 83 p. 100), plus rarement sur des plantes au stade 3 feuilles (17 p. 100).
L'intervalle de temps qui sépare l'émergence du coléoptile hors du sol de l'apparition de la $1^{\text {re }}$ talle est très variable comme le montre la figure 1.

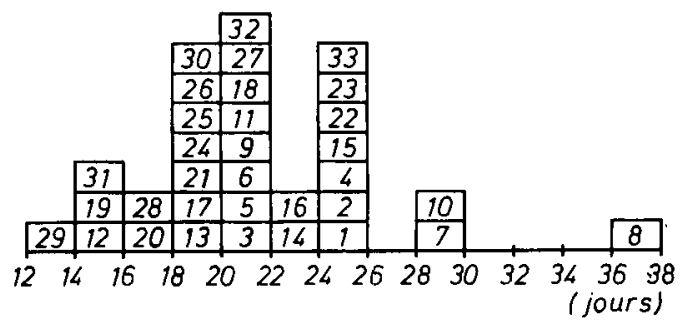

Figure 1

Histogramme des durées de la phase levée-début tallage (les nombres indiqués correspondent aux numéros de semis, cf. tabl.1)

Histogram of time from emergence- to tillering (for significance of numbers, see table 1).

Les durées de la phase levée-début tallage s'étalent de 12 à $36 \mathrm{j}$, pour une moyenne générale de 20,9 . Les valeurs les plus fréquemment rencontrées sont comprises entre 18 et $26 \mathrm{j}$. On peut remarquer que les durées les plus courtes sont observées en fin de printemps-début été et les plus longues à l'automne.

\section{B. Analyse climatique des phases levée-début tallage}

Afin de caractériser les périodes climatiques correspondant aux phases levée-début tallage, nous avons réalisé l'analyse en composantes principales du tableau des paramètres climatiques moyens sur chacune de ces périodes, qui donne une vue plus synthétique que la juxtaposition de polygones de fréquences.

Le tableau 2 donne les caractéristiques de l'analyse relatives aux 5 composantes.

Les 2 premiers axes extraient donc un pourcentage de variance du tableau initial égal à 85,9 p. 100 .

Le $1^{\text {er }}$ axe est dû en majeure partie aux températures et au déficit hydrique théorique, en opposition évidente avec l'humidité du sol. Le rayonnement global y apparaît dans une moindre mesure et est mieux représenté sur le $2^{\mathrm{e}}$ axe, sur lequel apparaît également la température de l'air.

La structure des variables montre donc comme principale source de variabilité un complexe eau-température et une source de variabilité secondaire due en partie au rayonnement.

La figure 2 présente la projection des périodes levéedébut tallage dans le plan des 2 premiers axes factoriels. Il y apparaît que l'axe 1 discrimine les différentes années alors que l'axe 2 discrimine les différentes périodes de culture 
TABLEAU 2

Corrélations des variables climatiques avec leurs composantes principales.

Correlations between climatic factors and their principal components.

\begin{tabular}{|c|c|c|c|c|c|}
\hline $\mathrm{N}^{\circ}$ de l'axe & 1 & 2 & 3 & 4 & 5 \\
\hline Rayonnement global & 0,609 & 0,758 & 0,194 & $-0,110$ & 0,068 \\
\hline Tempćrature air & 0,801 & $-0,475$ & 0,333 & 0,092 & 0,111 \\
\hline Température sol & 0,957 & -0.074 & 0,213 & $-0,082$ & $-0,166$ \\
\hline Humidité sol & $-0,851$ & 0,203 & 0,397 & 0,274 & $-0,045$ \\
\hline Déficit hydrique & 0,872 & 0,187 & $-0,288$ & 0,349 & $-0,011$ \\
\hline Pourcentage d'incrtic extrait & 68,23 & 17,66 & 8,70 & 4,48 & 0,93 \\
\hline
\end{tabular}

d'une même année. La variabilité principale semble donc due à une différence entre années chaudes et sèches et années humides, notamment sensible sur les phases de fin de printemps et de début d'été. La variabilité secondaire serait alors due à une différence entre périodes printanières, où le rayonnement global est plus fort, et périodes estivales et automnales, au rayonnement comparativement plus faible et à température de l'air plus forte, ce qui correspond au décalage classiquement observé entre la variation du rayonnement et celle de la température au cours de l'annéc.

Cette analyse indique de façon synthétique que les phases « levée-début tallage » que nous avons étudiées se déroulent sous l'influence de situations climatiques très diverses, tant par les vitesses d'évolution que par les distributions des paramètres climatiques en jeu. Pratiquement, tous les cas d'espèces sont représentés, du moins en ce qui concerne les conditions normales de culture (on ne sème pas de ray-grass d'Italie pendant les mois d'hiver).

\section{TABLEAU 3}

Corrélation de la vitesse de déclenchement du tallage avec les composantes principales de l'analyse climatique.

Emergence-tillering rate in correlation with principal components of climatic factors.

\begin{tabular}{|c|c|c|c|c|c|}
\hline $\mathrm{N}^{0}$ de l'axe & 1 & 2 & 3 & 4 & 5 \\
\hline Coefficient de corrélation & 0,66 & 0,14 & 0,29 & $-0,05$ & 0,08 \\
\hline
\end{tabular}

\section{Choix d'un critère biologique}

Le choix du critère biologique caractéristique de la phase levée-début tallage s'est porté sur la vitesse d'entrée en tallage, égale à l'inverse de la durée séparant les 2 stades.

Nous avons supposé que la loi d'action des facteurs climatiques sur cette vitesse est linéaire dans l'intervalle de variation correspondant aux fluctuations normales du climat, sur le site d'expérience (climat océanique, dans lequel les amplitudes de variation des facteurs sont faibles). Pour une gamme de variation plus large (climat plus continental), cette approximation serait insuffisante car l'on sait que, dans ce cas, la loi d'action peut revêtir diverses formes : loi en cloche (de Parcevaux, 1967 ; Durand, 1967 ; Simon, $1981 a$ ), loi du minimum, loi du tout ou rien, etc... Compte tenu des valeurs effectivement prises par les différents paramètres, l'approximation faite semble acceptable.

\section{Les facteurs climatiques actifs sur le déclenchement du tallage}

On a choisi comme variables explicatives de la vitesse de déclenchement du tallage les variables climatiques analysées au paragraphe $B$.

Le choix d'une combinaison linéaire de ces variables comme modèle explicatif se justifie par l'examen du tableau 3, qui présente les coefficients de corrélation de la vitesse de déclenchement du tallage avec les axes successifs extraits par l'analyse en composantes principales des facteurs climatiques. On constate que la vitesse de déclenchement est mieux corrélée avec le premier axe et donc avec la combinaison linéaire des variables climatiques qui est la mieux échantillonnée: le modèle proposé semble donc pertinent et ne paraît pas oublier de variable fondamentale.

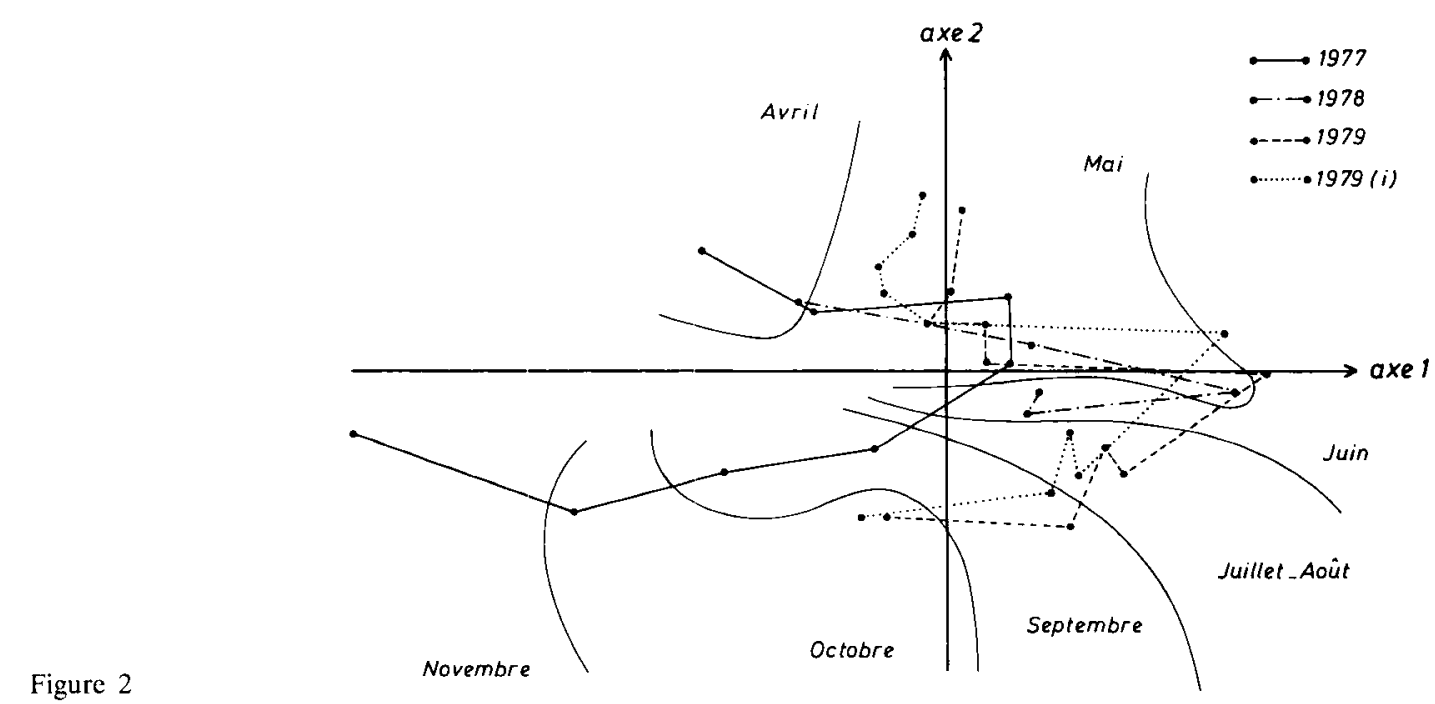

Figure 2 levée-début tallage sur le plan des 2 premiers axes factoriels.

Projection of the first two principal components for the emergencetillering climatic periods. 
Le tableau 4 montre les coefficients de corrélation des variables climatiques explicatives entre elles. On remarque les fortes corrélations entre températures de l'air et du sol ainsi qu'entre température du sol, humidité du sol et déficit hydrique. Cette constatation justifie l'emploi de la «ridge regression » comparativement à la régression multiple classique.

\section{TABLEAU 4}

Matrice de corrélation des variables explicatives. Correlation matrix of predictors.

\begin{tabular}{lrrrrr}
\hline \hline & $\mathrm{Rg}$ & $\mathrm{Ta}$ & $\mathrm{Ts}$ & $\mathrm{Hs}$ & $\mathrm{Dh}$ \\
\hline $\mathrm{Rg}$ & 1,000 & & & & \\
$\mathrm{Ta}$ & 0,189 & 1,000 & & & \\
Ts & 0,564 & 0,846 & 1,000 & & \\
$\mathrm{Hs}$ & $-0,319$ & $-0,625$ & $-0,759$ & 1,000 & \\
Dh & 0,577 & 0,544 & 0,732 & $-0,722$ & 1,000 \\
\hline \hline
\end{tabular}

La figure 3 présente les valeurs des VIF en fonction des différentes introductions de variables pour la régression classique. Si l'on fixe la limite supérieure «tolérable »du VIF à 2, on voit que seuls le rayonnement global et la température du sol peuvent être retenus. Ceci est confirmé par le tableau 5 où l'on trouve les coefficients de régression des variables climatiques dans le cas d'un modèle à 2 variables et dans celui d'un modèle complet (à 5 variables). On constate que le modèle à 2 variables choisit le rayonnement global et la température du sol, alors que le modèle complet donne la prééminence au rayonnement global et à la température de l'air, d'où un manque de logique entre les 2 modèles.

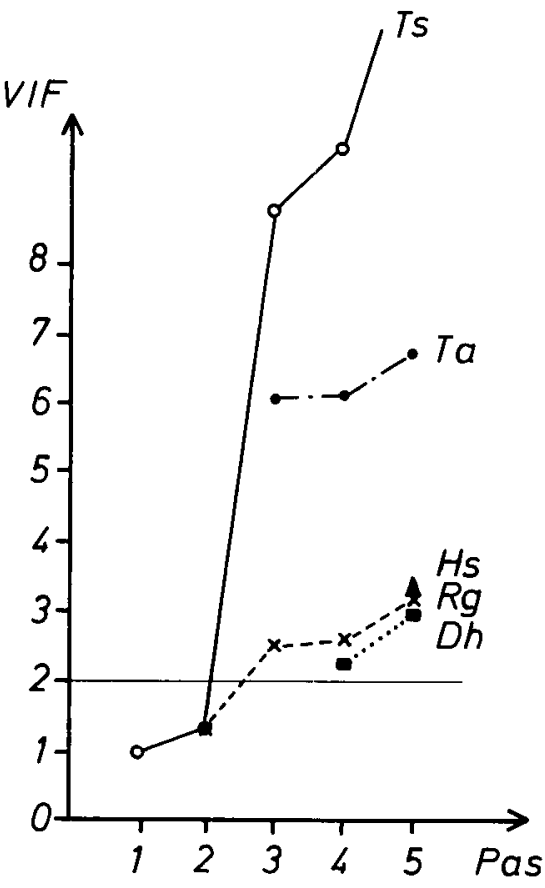

Figure 3

Evolution du coefficient V.I.F. en fonction du nombre de variables introduites.

Variance inflation factor in relation with the number of variables introduced.

La figure 4 présente, pour le modèle complet, l'évolution des coefficients des variables centrées, en fonction de différentes valeurs du coefficient $\mathrm{k}$ de la ridge regression. L'examen de cette «trace ridge » montre que les coefficients de régression se stabilisent pour une valeur de $k$

TABLEAU 5

Résultats de la régression multiple non biaisée $(k=0)$ et de la ridge regression $(k=0,1)$. Unbiased multiple regression $(k=0)$ and ridge regression $(k=0.1)$ results.

\begin{tabular}{|c|c|c|c|c|}
\hline & & \multicolumn{2}{|c|}{$\begin{array}{l}\text { Régression multiple non biaisée } \\
\qquad \mathbf{k}=0\end{array}$} & \multirow{2}{*}{$\begin{array}{c}\begin{array}{c}\text { Ridge regression } \\
\mathrm{k}=0.1\end{array} \\
5\end{array}$} \\
\hline Nombre de variables pr & npte & 2 & 5 & \\
\hline $\begin{array}{l}\text { Cocfficients des variables } \\
\text { centrées réduites }\end{array}$ & $\begin{array}{l}\mathrm{Rg} \\
\mathrm{Ta} \\
\mathrm{Ts} \\
\mathrm{Hs} \\
\mathrm{Dh}\end{array}$ & $\begin{array}{l}0,23 \\
0,55^{* *}\end{array}$ & $\begin{array}{r}0,47 \\
0,52 \\
0,04 \\
0,00 \\
-0,08\end{array}$ & $\begin{array}{r}0,34 \\
0,34 \\
0,20 \\
0,02 \\
-0,02\end{array}$ \\
\hline $\begin{array}{l}\text { Coefficients des variables } \\
\text { non centrées }\end{array}$ & $\begin{array}{c}\mathrm{Rg} \\
\mathrm{Ta} \\
\mathrm{Ts} \\
\mathrm{Hs} \\
\text { Dh } \\
\text { Constante }\end{array}$ & $\begin{array}{c}0,23 \cdot 10^{-4} \\
0,21 \cdot 10^{-2 * *} \\
0,01\end{array}$ & $\begin{array}{c}0,46 \cdot 10^{-4} \\
0,32 \cdot 10^{-2} \\
0,15 \cdot 10^{-3} \\
0,11 \cdot 10^{-4} \\
0,22 \cdot 10^{-4} \\
0,01\end{array}$ & $\begin{array}{c}0,34 \cdot 10^{-4} \\
0,21 \cdot 10^{-2} \\
0,80 \cdot 10^{-3} \\
0,85 \cdot 10^{-4} \\
-0,60 \cdot 10^{-5} \\
-0,35 \cdot 10^{-2}\end{array}$ \\
\hline $\begin{array}{l}\text { Test de la signification } \\
\text { de l'ajustement }\end{array}$ & $\begin{array}{l}F(5,27) \\
F(2,30)\end{array}$ & $14,95 * * *$ & $6,49 * *$ & $5,65 * *$ \\
\hline $\begin{array}{l}\text { Pourcentage de variance } \\
\text { expliquée }\end{array}$ & $\left(\mathrm{R}^{2}\right) * 100$ & 49,8 & 54,6 & 48,3 \\
\hline
\end{tabular}




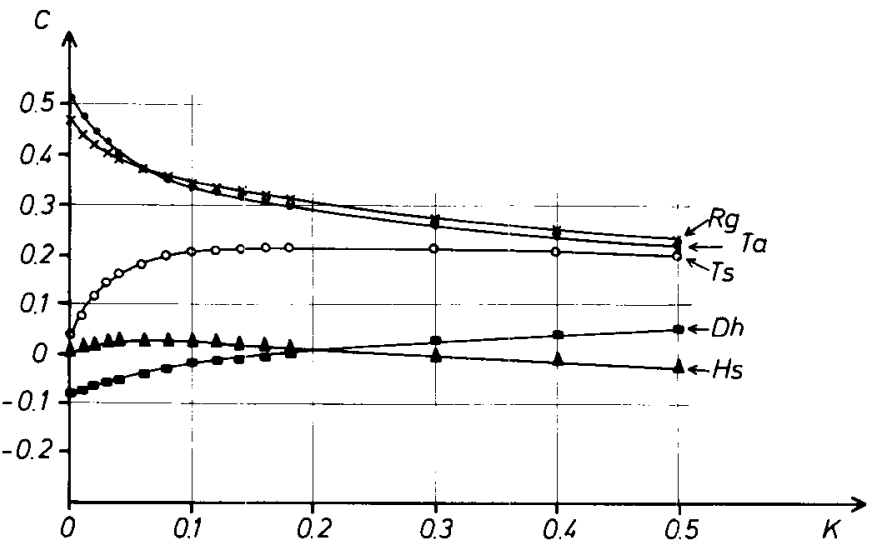

Figure 4

Valeurs prises par les coefficients de régression des variables centrées $(c)$ pour différentes valeurs de $k$.

Ridge trace (c) for several values of $k$.

grossièrement égale à 0,1 . Les coefficients de régression relatifs au modèle complet pour cette valeur de $\mathrm{k}$ sont regroupés dans le tableau 5 , où l'on constate que le rayonnement global et les températures de l'air et du sol sont affectés de poids du même ordre de grandeur, ce qui est cohérent avec, d'une part, le modèle à 2 variables de la régression classique et, d'autre part, la corrélation entre température de l'air et température du sol. Il apparaît donc que les facteurs importants sont le rayonnement global et la température, que celle-ci soit température de l'air ou du sol, ou combinaison des deux.

Enfin, la figure 5 présente, pour les modèles complets, la relation entre résidus de la régression et valeurs estimées. On y constate, dans les 2 cas, une augmentation de la variabilité des résidus avec l'ordre de grandeur des valeurs estimées par les modèles examinés. Ceci traduit vraisemblablement l'augmentation de la variabilité de la vitesse de levée pour les fortes valeurs des facteurs explicatifs, due à l'hétérogénéité génétique du matériel végétal.

\section{E. Exigences thermiques du ray-grass d'Italie pour la phase levée-début tallage}

Ayant montré, dans ce qui précède, le rôle déterminant de la température sur le déroulement de la phase levée- début tallage du ray-grass d'Italie (qu'il s'agisse de la température de l'air ou de la température du sol), il peut être intéressant de fixer les ordres de grandeur des besoins thermiques nécessaires à l'accomplissement de cette phase.

La méthode employée est celle des sommes de températures exposée par DURAND (1967), en dépit des réserves qui peuvent être formulées vis-à-vis de cette méthode. Cette dernière, rappclons-le, repose sur la linéarité de relation entre vitesse de développement et température. La somme des températures moyennes journalières, diminuées d'un seuil $\mathrm{T}_{0}$, est constante pour une phase de développement donnée :

$$
\sum\left(\mathrm{T}-\mathrm{T}_{0}\right)=\mathrm{K}
$$

Nous éliminerons de ce calcul les phases:

- où le facteur hydrique est légèrement limitant (les phases conservées présentent des humidités de sol situées dans une gamme correspondant à la réserve facilement utilisable, soit $H_{s}>13$ p. 100),

- où les températures extrêmes journalières (soit de l'air, soit du sol) ne sont pas situées dans un intervalle où l'hypothèse de linćarité de relation est acceptable (soit les valcurs inférieures à $0{ }^{\circ} \mathrm{C}$ et supérieures à $25^{\circ} \mathrm{C}$ ).

Restent alors 17 traitements pour les températures de l'air et 17 traitements pour les températures du sol, 15 étant communs.

Les résultats de ces calculs sont présentés à la figure 6 .

Le scuil thermique apparent, défini par la méthode du coefficient de variation minimum (BLOC \& GOUET, 1973), cst voisin de $0^{\circ} \mathrm{C}$ dans les 2 cas. Un intervalle de confiance autour du coefficient de variation minimum (CRAMER, 1971 ) indique en fait que ce seuil est compris entre 0 et $6^{\circ} \mathrm{C}$ pour les températures de l'air et 0 et $3{ }^{\circ} \mathrm{C}$ pour les températures du sol.

L'observation des sommes de températures nécessaires à l'accomplissement de la phase levée-début tallage du raygrass d'Italie confirme le poids équivalent des températures de l'air et de celles du sol sur le déroulement de cette phase : pour un seuil de $0^{\circ} \mathrm{C}$, les sommes de températures sont respectivement de $284^{\circ} \mathrm{C}$. j pour les températures du sol et de $265^{\circ} \mathrm{C}$. j pour celles de l'air, valeurs non significativement différentes.
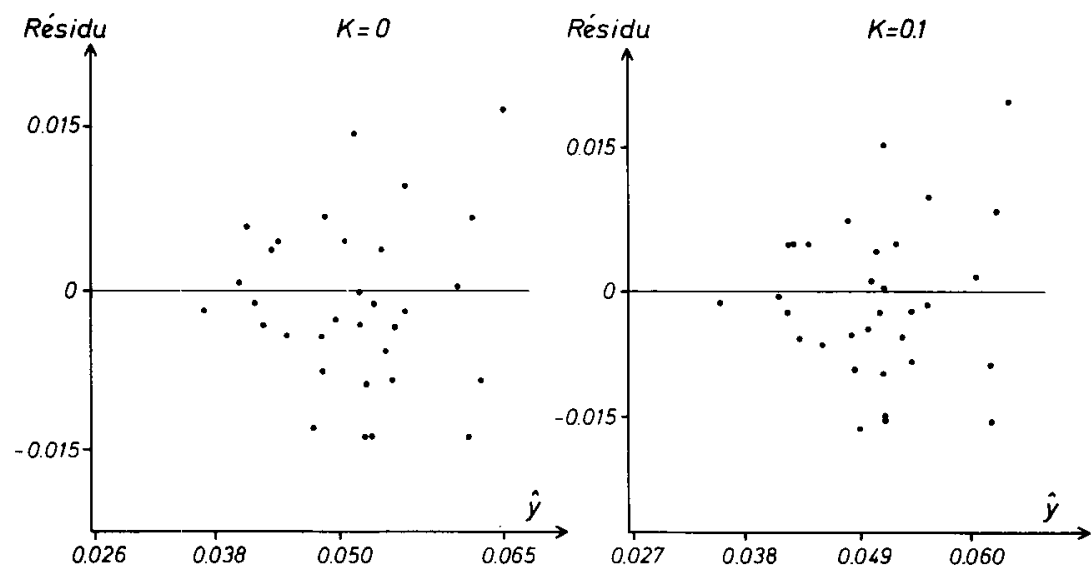


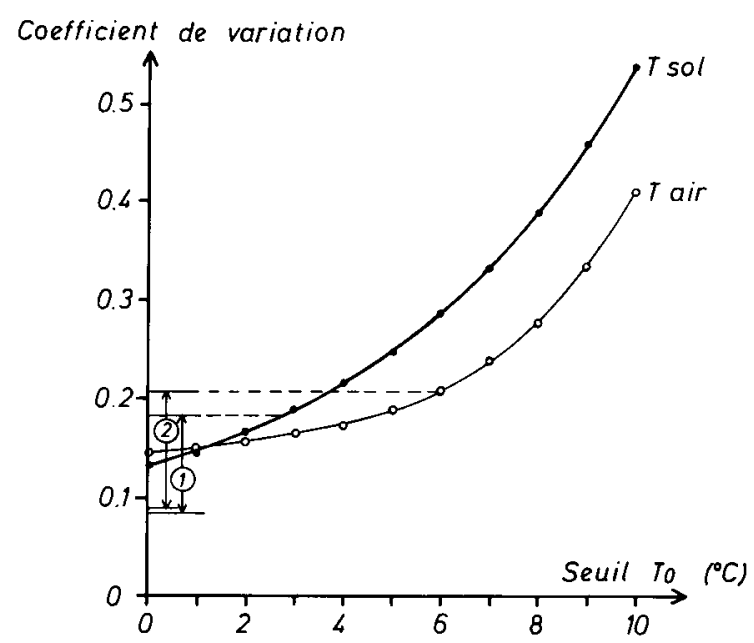

Figure 6

Evolution des coefficients de variation des sommes des températures de l'air $(\grave{a} l, 50 \mathrm{~m})$ et du sol $(\grave{a}-0,10 \mathrm{~m})$ en fonction du seuil apparent $T_{0}$.

(1) : intervalle de confiance du c.v. pour la température du sol et pour $T_{0}=0{ }^{\circ} \mathrm{C}$.

(2) : id. pour la température de l'air.

Coefficient of variation of temperature sums from daily temperature means in air $(1.50 \mathrm{~m})$ and soil $(-0.10 \mathrm{~m})$ in relation to the base temperature selected

(1) : confidence interval of c.v. for soil temperature and for $T_{0}=0{ }^{\circ} \mathrm{C}$ (2) : the same for air temperature.

\section{DISCUSSION}

\section{A. Nature des facteurs actifs}

Deux facteurs actifs ont été mis précédemment en évidence: la température et le rayonnement global. Les travaux antérieurs sur graminées fourragères ( $\S$ II) qui montrent lincidence du facteur thermique sur le phyllochrone et du facteur rayonnement sur l'élongation des talles sont confirmés par les résultats de notre étude. Le fait que ce soit la température de l'air ou du sol qui explique le mieux la durée de la phase «levée-début tallage » résulte principalement du fait que c'est la durée d'émission des premières feuilles qui occupe la plus grande partie de cette phase, la durée relative à la croissance de la $1^{\text {rc }}$ talle étant comparativement brève.

\section{B. Validité des modèles proposés}

Bien que le choix des variables explicatives semble pertinent ( $\S$ IV D), quel que soit le modèle retenu (régression multiple avec 2 facteurs explicatifs, ou ridge regression avec l'ensemble des facteurs), la variabilité expliquée n'est que de 50 p. 100 . Cela signifie que d'autres facteurs, non pris en compte, sont également en jeu. Parmi ceux-ci, il est probable que le plus important soit la nature génétique du matériel utilisé. Les cultivars de ray-grass d'Italie sont des variétés de populations de variabilité génétique très importante. La réponse à un facteur du milieu des différents individus constituant la population est, de ce fait, très hétérogène.

Notons que d'autres sources de variation difficilement contrôlables pourraient également être envisagées: les variations microlocales des facteurs explicatifs, l'alimentation azotée des plantes, etc.

D'un point de vue méthodologique, l'utilisation de la ridge regression conduit à un modèle complet plus cohérent que la régression classique à partir de variables expličatives très corrélées. Mais cela ne suffit pas à pallier un autre handicap des études portant sur du matériel génétique hétérogène, la variabilité de réponse de la plante crô̂t lorsque les valeurs des facteurs climatiques augmentent, ceci rend sujette à caution l'utilisation du modèle linéaire.

\section{Exigences thermiques}

Contrairement à ce qui a pu être dit pour la phase semislevée de la même plante (SIMON, 1981b), l'étude des coefficients de variation respectifs montre que les sommes de températures du sol (c.v. $=0,13$ ) caractérisent mieux la phase levée-début tallage que le nombre de jours calendaires (c.v. $=0,22)$. Ceci n'est pas vrai pour les températures de l'air (c.v. $=0,15$ contre 0,17 pour le nombre de jours). Il semble donc plus intéressant d'utiliser la température du sol pour caler la date de début tallage.

\section{CONCLUSION}

L'étude en conditions naturelles du déclenchement du tallage chez le ray-grass d'Italie souligne les difficultés de la recherche statistique de relations plante-climat dans le cas d'une variété de nature génétique hétérogène.

Ainsi, les liaisons entre facteurs climatiques obligent à une grande prudence dans la prise en compte des variables explicatives (examen des VIFs, utilisation de la ridge regression, examen des résidus du modèle).

On a cependant pu retrouver les résultats bibliographiques, obtenus pour la plupart en conditions contrôlées, mettre en évidence l'influence de la température (qu'elle soit de l'air ou du sol) et du rayonnement global (bien qu'avec un poids moindre) et fixer les normes écologiques relatives à cette phase : en ce qui concerne la température $\mathrm{du}$ sol, sous réserve que celle-ci soit inférieure à $25^{\circ} \mathrm{C}$, et en l'absence de facteur hydrique limitant, la phase levée-début tallage peut être caractérisée par une somme de températures de $280^{\circ} \mathrm{C}$.j pour un seuil apparent de $0{ }^{\circ} \mathrm{C}$.

Reçu le 17 juin 1982 Accepté le 3 mars 1983.

\section{RÉFÉRENCES BIBLIOGRAPHIQUES}

Auda H., Blaser R. E., Brown R. H., 1966. Tillering and carbohydrate contents of orchard grass as influenced by environmental factors. Crop Sci., 6 (2), 139-143.

Beevers L., Edwards K. J. R., 1960. Biochemical variation. Welsh Plant Breed. Stn. Rep., 19-21.
Bloc D., Gouet J. P., 1973. Influence des sommes de températures sur la maturité du maïs. Publication I.T.C.F.-A.G.P.M., 13 p.

Bommer D., 1962. Expériences sur la physiologie du tallage chez les graminées pérennes. Annu. Meet. West. Soc. Crop. Sci. Bozeman, Montana, $6 \mathrm{p}$. 
Cooper J. P., 1964. Climatic variation in forage grasses. I. Leaf development in climatic races of Lolium and Dactylis. J. appl. Ecol., 1, 45-61.

Cramer H., 1971. Mathematical methods of statistics. Princeton University Press, 575 p.

De Parcevaux S., Durand R., Roche P., 1967, Action de la température sur la croissance et le développement du lin. Ann. Physiol. veg., 9 (1), 87-105.

Draper N. R., Van Nostrand R. C., 1979. Ridge regression and James Stein estimation : review and comments. Technometrics, 21 (4), 451-466.

Duby C., Masson J. P., 1980. Conséquences de la non-orthogonalité en régression. Biom. Praxim, 20 (3/4), 59-78.

Durand R., 1967. Action de la température et du rayonnement sur la croissance. Ann. Physiol. vég., 9 (1), 5-27.

Friend D. J. C., Fisher J. E., Nelson V. A., 1962. Leaf growth of Marquis wheat as regulated by temperature, light intensities and day length. Can. J. Bot., 40, 1299-1311.

Gibbons D., 1981. A simulation study of some ridge estimators. $J$. am. Stat. Assoc., 76 (373), 131-139.
Gillet M., 1970. Sur quelques aspects de la croissance et du développement de la plante entière de graminée en conditions naturelles: Festuca pratensis Huds. Thèse, Poitiers, $190 \mathrm{p}$.

Gillet M., 1980. Les graminées fourragères. Collection « Nature et Agriculture », Gauthiers-Villars, 306 p.

Hoerl A. E., 1962. Application of ridge analysis to regression problems. Chem. Eng. Prog., 58, 54-59.

Hoerl A. E., Kennard R. W., 1970. Ridge regression: biased estimation for non-orthogonal problems. Technometrics, 12, 69-82.

Katz R. W., 1979. Sensitivity analysis of statistical crop-weather models. Agric. Meteorol., 20, 291-300.

Marquardt D. W., Snee R. D., 1975. Ridge regression in practice. Am. Stat. 29 (1), 3-20.

Simon J. C., 1979. Trente années de mesures au poste météorologique de Quimper. Note Interne, Agronomie Quimper, 79/2, $10 \mathrm{p}$. Simon J. C., 1981a. Contribution à l'étude écophysiologique de la phase semis-levée du ray-grass d'Italie. I. Etude en conditions contrôlées. Agronomie, 1 (5), 339-344.

Simon J. C., 1981b. Contribution à l'étude écophysiologique de la phase semis-levée du ray-grass d'Italie. II. Etude en conditions naturelles. Agronomie, 1 (5), 345-354. 